\title{
Wheelchair Users' Satisfaction with the Prescribed Wheelchairs and Wheelchair Services in Croatia
}

\author{
Siniša Štefanac ${ }^{1,2}$, Igor Grabovac ${ }^{\mathbf{2}}$ and Sofi Fristedt ${ }^{4}$ \\ ${ }^{1}$ Ludwig Boltzmann Society - Rheumatoid Cluster for Arthritis and Rehabilitation, Vienna, Austria \\ ${ }^{2}$ Institute for Outcomes Research, Centre for Medical Statistics, Informatics and Intelligent Systems, Medical university \\ of Vienna, Austria \\ ${ }^{3}$ Department of Social and Preventive Medicine, Center for Public Health, Medical University of Vienna, Vienna, Austria \\ ${ }^{4}$ ARN-J Aging Research Network - Jönköping, Department of Rehabilitation, Jönköping University, School of Health \\ and Welfare, Sweden
}

\begin{abstract}
A B S T R A C T
The authors tried to determine the wheelchair users' satisfaction with prescribed wheelchairs and wheelchair services in Croatia. An online survey was distributed among members of the Croatian Paraplegic and Tetraplegic Alliance. One hundred-four participants were included in the analyses using non-parametric statistics. Most participants were satisfied with wheelchair dimensions $(n=84 ; 81 \%)$, functionality $(n=80 ; 77 \%)$, easiness to use $(n=81 ; 78 \%)$, comfort $(n=69 ; 66 \%)$ and wheelchair parts adjustment $(n=64 ; 62 \%)$. Individuals satisfied with wheelchair characteristics were also more engaged in the activities of everyday living than participants who were not satisfied. Significant activity participation was found in the following activities: bathing/showering $\left(x^{2}=7.02 ; d f=1 ; p=0.017\right)$, transfers $\left(x^{2}=8.49 ; d f=1 ; p=0.01\right)$, bladder/bowel management $\left(x^{2}=17.77 ; d f=1 ; p<0.001\right)$, food preparation $\left(x^{2}=8.34 ; d f=1 ; p=0.004\right)$, eating $\left(x^{2}=9.33 ; d f=1 ; p=0.014\right)$ and doing household chores $\left(x^{2}=13.28 ; d f=1 ; p<0.001\right)$. Fifty-seven participants $(54.8 \%)$ had received wheelchair assessment and were also predominantly satisfied with the assessment ( $n=51 ; 90 \%)$, selection process $(n=49 ; 79 \%)$, equipment trial ( $n=24 ; 80 \%)$, education/skills training $(n=40 ; 89 \%)$ and the fitting process $(n=38 ; 86 \%)$. Participants who received wheelchair assessment and skills training felt safer when using wheelchairs $\left(x^{2}=6.13 ; d f=1 ; p=0.013\right)$. Majority of the participants were unsure where to ask for second opinions regarding wheelchair assessment $(n=80 ; 77 \%)$ and where to seek wheelchair support groups ( $n=78 ; 75 \%)$. Satisfaction with wheelchairs and wheelchair services leads to more involvement in the activities of everyday living and increases feelings of safety.
\end{abstract}

Key words: activities of everyday living; occupational therapy; wheelchair prescription; wheelchair satisfaction; wheelchair services

\section{Introduction}

Wheelchairs support mobility and thereby health and well-being from participation in important activities of everyday living at home and in the community. Consequently, inaccuracies in wheelchair prescription, as well as their disuse may lead to participation restrictions due to poor posture, increased energy consumption, development of contractures/deformities and put the user at a risk of other complications such as respiration, communication and swallowing difficulties but also urinary and digestive infections, development of pressure ulcers, as well as pain $^{1-6}$. Psychosocial implications may also occur, including feelings of stress, anxiety, depression, hopelessness, poor self-esteem, which may lead to verbal and/or physical hostility as well as difficulties with social support. This may in turn negatively affect community integration and social participation.

To avoid complications, wheelchair assessment should be carried out by a multidisciplinary team with appropriate knowledge in posture management and mobility, including occupational therapists, physiotherapists, technicians and rehabilitation engineers together with active participation from the users and caregivers ${ }^{1,2,7-9}$. Ideally, wheelchair assessment should be done at wheelchair specialist centres with appropriate transfer and wheelchair equipment (hoists, sliding sheets, etc.) as well as different types/ranges of wheelchairs on disposal. When relevant, the wheelchair assessment may be carried out in the users' own homes or domiciliary locations (e.g. schools or other), especially if environmental factors are crucial for a wheel- 
chair assessment and prescription ${ }^{9}$. Providing users with wheelchairs is not enough, as this process should encompass several stages including wheelchair assessment and prescription, education on maintenance, skills training and regularly scheduled follow-ups.

Literature suggest that wheelchair assessment and prescription must encompass relevant medical information as well as a physical examination with detailed biomechanical/posture assessment, which includes seating, lying and standing positions including all fixed and correctable body parts and effects of various forces on posture, determination of functional ability and comfort levels $^{1,2,6,9-11}$. Each wheelchair prescription should be based on the user's goals in line with user's needs in their environment and lifestyle $e^{2,9,12-14}$. Setting up goals including time frames and follow-ups, planning and implementing an intervention and measuring the outcome are keystones for evidence-based practice ${ }^{15-19}$.

After the wheelchair assessment is done, users should be involved in the wheelchair selection process and trial proposed equipment. These processes should be done in a specialized wheelchair centre and then within the environment that the user inhabits. At this point it is imperative to determine the correct width, depth, height and other wheelchair features with the user in order to increase the comfort and safety levels of the user ${ }^{9,14,20-24}$.

As wheelchairs are more susceptible to damage from outside factors than most other mobility assistive devices, users need also to be coached about proper wheelchair use, hygiene and maintenance. Therefore, wheelchair users and their caregivers should receive proper training including wheelchair skill training, which should be done at home and other environments, as well as basic information on technical maintenance while not understating the need for regular professional maintenance appointments. This is vital as improper wheelchair use (reckless and speedy driving, bumping etc.) may have a negative influence on comfort and safety levels.

Finally, follow-up appointments are necessary in order to make sure that the wheelchair is in good condition, still fits the user and that the user still possesses the appropriate skill level. If needed regular follow-ups should also cover additional skills training. Unfortunately the described process is not always successful or followed and on average one-third, of all assistive technology devices are abandoned by users ${ }^{25}$. This may cause detrimental individual effects on the user, in terms of reduced quality of life, well-being and health outcomes respectively but also increased health care costs ${ }^{1,2,6,9,10,25-27}$. Wheelchair disuse should be a concern not only for users, but also for the health care professionals and community, and could be prevented by applying an evidence-based practice in the wheelchair selection process ${ }^{9-11}$.

Understanding wheelchair users' satisfaction levels may help occupational therapists and other health care professionals to increase wheelchair users' performance in the activities of everyday living, facilitate social and community participation and increase indoor/outdoor mobility, since it is more likely that the wheelchair users will use their wheelchairs if their satisfaction levels with the wheelchairs and wheelchair services are high ${ }^{17}$. Moreover, investigating the users' satisfaction level with their wheelchairs and wheelchair services may shed light on the quality of the whole wheelchair service process. Additional knowledge gaps relate to the lack of research on wheelchair users' satisfaction with the wheelchair characteristics and wheelchair services and its relationship to involvement in the activities of everyday living.

Therefore, the purpose of the present study was to determine the satisfaction levels with the current wheelchair as well as of wheelchair assessment and selection process, trial equipment and wheelchair education/skills training. An additional purpose was to determine the relationship between the wheelchair users' satisfaction levels and participation in the activities of everyday living.

\section{Materials and Methods}

\section{Design}

This study was designed as a cross-sectional Internet based study with adult wheelchair users in Croatia. The study was done in accordance with the Helsinki Declaration and Croatian legal statutes. Approval to contact was obtained on the $20^{\text {th }}$ January 2017 from the Board of the Croatian Paraplegic and Tetraplegic Alliance in Croatia. Inclusion criteria for this study were: wheelchair users who were 18 years of age or older, with any form of physical disability lasting at least one year, using any manual or powered wheelchairs for at least 3 months after the injury/disability, able to operate their wheelchair independently or with minimum to moderate help and sufficient knowledge of the Croatian language.

\section{Participants}

Members of the Croatian Paraplegic and Tetraplegic Alliance were asked to participate via a link to an online survey platform that was sent to them through the online mailing list. Information about the study was supplied on the first page of the survey. This information underlined the voluntary nature of participation, as well as guaranteed the confidential handling of the data. The participants gave their informed consent by choosing an option allowing access to the questionnaire. At any given point, the participants were allowed to withdraw from the study without having to state a reason. The email address of the project leader was provided during the entire survey for the participants to contact if any questions arise.

A total number of 115 participants responded to the questionnaire. Out of these, two did not use wheelchair anymore and nine participants were unable to operate their wheelchairs independently. Consequently, 104 participants were included in the statistical analyses.

\section{Methods and questionnaire}

For the purposes of this study a self-administered questionnaire comprising of 80 items in three parts was 
TABLE 1

SAMPLE CHARACTERISTICS (N=104)

\begin{tabular}{lc}
\hline Variable & $\begin{array}{c}\text { Frequencies } \\
\text { n (\%) }\end{array}$ \\
\hline Age: Range, Mean (SD) & $20-72,42.98(11.3)$ \\
\hline Gender: Male/Female & $70(67.3) / 34(32.7)$ \\
\hline Primary cause of disability: Spinal cord injury / Other & $94(90.4) / 10(9.6)$ \\
\hline Time lived with disability: & $26(25) / 26(25)$ \\
- 1-4 years / 5-8 years & $52(50)$ \\
- 9 or more years & \\
\hline Place of residence: & $40(38.5)$ \\
- Zagreb (the capital city of Croatia) & $23(22.1)$ \\
- Big city (from 50.001 to 500.000 inhabitants) & $19(18.3)$ \\
- City (from 10.001 to 50.000 inhabitants) & $9(8.7)$ \\
- Smaller town (from 5001 to 10.000 inhabitants) & $13(12.5)$ \\
- Village (of up to 5000 inhabitants) & \\
\hline Housing: & $60(57.7) / 43(41.3)$ \\
- Apartment / House & $1(1)$ \\
\hline
\end{tabular}

Educational level:

- University degree or higher / College education

$19(18.3) / 17(16.3)$

- High school / Primary school or unskilled

$63(60.6) / 5(4.8)$

\section{Household incomes:}

- No income / Up to 3000 Croatian Kuna*

$2(1.9) / 26(25)$

- Between 3001 and 5000 Croatian Kuna

- Between 5001 and 8000 Croatian Kuna

$26(25)$

- More than 8000 Croatian Kuna

$17(16.3)$

\section{Partnership status:}

- Live / Do not live with a spouse or a partner

\section{Employment:}

- Employed (paid work) / Unemployed

$22(21.2) / 36(34.6)$

- Retired / Volunteering

39 (37.5) / 2 (1.9)

- Attending school, college or other educational activities

$5(4.8)$

Main mode of community mobility:

- Independently drive wheelchair not using other modes

- Drive an accessible car / Other people drive me in their car

- Only by organised accessible car/van (e.g. from association)

$1(1)$

- Combinations of public transportation, ambulance and association car

$90(86.5)$

\section{Type of wheelchair used:}

- Manual / Electric

$86(82.6) / 8(7.7)$

- Manual and electric

- Power-assisted

$1(1)$

\section{Who financed the wheelchair:}

- Personal / Croatian Health Insurance Fund

$11(10.6) / 42(40.4)$

- Donation / Combinations of all of the above

$10(9.6) / 41(39.4)$

\section{Duration of using a wheelchair:}

-3 months to 1 year

- 1-2 years / 2-3 years

$4(3.8) / 10(9.6)$

$-4-5$ years / 5 or more years

$13(12.5) / 73(70.2)$ 


\begin{tabular}{lc}
\hline Hours spent in a wheelchair / day: & \\
- Up to 5 hours & $5(4.9)$ \\
-6-8 hours / 9-12 hours & $27(26) / 38(36.5)$ \\
- 13-18 hours / 19 hours or more & $30(28.8) / 4(3.8)$ \\
\hline Wheelchair mobility: Independent/Dependent** & $98(94.2) / 6(5.8)$ \\
\hline Ever injured yourself when using a wheelchair: Yes/No & $60(57.7) / 44(42.3)$ \\
\hline Feeling safe when using a wheelchair: Yes/No & $67(64.4) / 37(35.6)$ \\
\hline Reasons for not feeling safe when using a wheelchair (n=37): & $24(64.9)$ \\
- Heavy wheelchair & $20(54.1) / 21(56.8)$ \\
- Sliding down in wheelchair / Slippery upholstery & $10(27)$ \\
- Improper positioning in a wheelchair & $26(70.3)$ \\
- Not adjusted or missing wheelchair accessory (e.g. belt) & $12(32.4)$ \\
- Wheelchair parts not functioning properly (e.g. brakes) & $10(27)$ \\
- Broken, wrongly positioned or missing anti-tippers & $7(18.9)$ / 12 (32.4) \\
- Worn casters and/or rear wheels / Worn wheelchair & $17(16.3)$ \\
- Dimensions of wheelchair not individually fitted & $5(13.5) / 4(10.8)$ \\
- Rear wheels / Casters not optimally positioned for me & $3(8.1) / 3(8.1)$ \\
\hline - Bent/twisted wheelchair / Improper push-handles height & \\
\hline *1 Croatian Kuna equals to 0,13 Euros & \\
** Requires minimum to moderate help &
\end{tabular}

created. The questionnaire consisted of single and multiple choice and "skip-logic questions" that guided the respondents throughout the cross-sectional survey. The questionnaire took about 15 minutes to complete and it was possible to pause it and return to the last answered question as many times as needed. After the respondents answered the questions, the answers were stored on the server (https://www.soscisurvey.de) without personal data of respondents, to preserve confidentiality. The link of the questionnaire was active for 35 days, from February $13^{\text {th }}$ until March $20^{\text {th }} 2017$.

\section{Part 1- Current Wheelchair}

The first part of survey consisted of 60 questions on wheelchair user's satisfaction with the current wheelchair and covered questions on past wheelchair assessments and selection, trial equipment experience, fitting and delivery, education and skills training, safety, maintenance and follow-ups. Also, the participants were asked about availability of wheelchair services, wheelchair assessments, mutual wheelchair support groups, professional advice and repairs/maintenance in their local community.

\section{Part 2-Activities of Daily Living}

The second part consisted of eight questions on activities of everyday living performance (e.g. independence, habits, expectations from others), as well as the level of significance of different activities. Moreover, questions on physical accessibility at home or in community was also included.

\section{Part 3 - Sociodemographic Data}

The last part of the questionnaire included twelve questions on sociodemographic information, such as gender, age, primary cause of disability, duration of disability, education level, partnership status, employment, monthly income and place of residence, which were used for further analyses and sample description.

\section{Statistical analyses}

Mean values and standard deviations were identified in the case of normal distribution, and qualitative variables were reported as frequency, percentage, median and range. Differences in frequency of categorical variables were determined by the $\mathrm{Chi}^{2}$-test for independence together with the Fisher's Exact Test and Bonferroni correction done. It was calculated using categorical, dichotomized and continuous variables where appropriate. All p-values below 0.05 were considered statistically significant. The statistical analyses were performed using the SPSS 21.0 statistical software.

\section{Results}

Considering demographics (Table 1), the participants were predominantly male and around 40 years of age. General wheelchair satisfaction characteristics in relation to the duration of wheelchair use are presented in table 2. Participants who were satisfied with the dimensions of their wheelchairs and who were feeling comfortable when using their wheelchairs were also more involved in the activities of everyday living as shown in table 3 .

\section{Wheelchair assessment}

More than half of the total number of participants $(n=57 ; 55 \%)$ were involved in the wheelchair assessment. Participants with whom the wheelchair assessment was done responded feeling safer when using their wheelchairs 
TABLE 2

WHEELCHAIR SATISFACTION (N=104)

\begin{tabular}{|c|c|c|c|}
\hline Wheelchair characteristics & $\begin{array}{c}\text { Using a wheelchair for } \\
\text { less than } 5 \text { years } \\
\text { Satisfied / Not satisfied } \\
\text { n (\%) }\end{array}$ & $\begin{array}{c}\text { Using a wheelchair for } \\
\text { more than } 5 \text { years } \\
\text { Satisfied / Not satisfied } \\
\text { n (\%) }\end{array}$ & $x^{2} ; d f ; p$-values \\
\hline Easiness to use a wheelchair & $26(25) / 5(4.8)$ & $55(52.9) / 18(17.3)$ & $\mathrm{X}^{2}=0.01 ; \mathrm{df}=1 ; \mathrm{p}=0.338$ \\
\hline Wheelchair comfort & $15(14.4) / 16(15.4)$ & $54(51.9)$ / 19 (18.3) & $X^{2}=6.38 ; d f=1 ; p=0.012$ \\
\hline Wheelchair dimensions (size, width, depth, height) & $28(26.9) / 3(2.9)$ & $56(53.8) / 17(16.3)$ & $\mathrm{x} 2=2.59 ; \mathrm{df}=1 ; \mathrm{p}=0.107$ \\
\hline Possibilities to adjust wheelchair parts & $15(14.4) / 16(15.4)$ & $49(47.1) / 24(23.1)$ & $\mathrm{X}^{2}=3.22 ; \mathrm{df}=1 ; \mathrm{p}=0.072$ \\
\hline Wheelchair functionality & $26(25) / 5(4.8)$ & $54(51.9) / 19(18.3)$ & $\mathrm{X}^{2}=1.28 ; \mathrm{df}=1 ; \mathrm{p}=0.273$ \\
\hline Wheelchair durability & $25(24) / 6(5.8)$ & $60(57.7) / 13(12.5)$ & $X^{2}=0.03 ; d f=1 ; p=0.852$ \\
\hline
\end{tabular}

when compared to the participants with whom the wheelchair assessment was not done $\left(\mathrm{n}=44 ; 77 \% ; \mathrm{X}^{2}=8.97 ; \mathrm{df}=1\right.$ $; p=0.003)$. The wheelchair assessment was mostly done by the non-medical professionals $(\mathrm{n}=36 ; 63 \%)$ such as wheelchair suppliers/vendors $(\mathrm{n}=32)$ and wheelchair technicians $(n=4)$. Medical professionals did the assessment in 21 cases (37\%), mostly by physiatrists/orthopaedic surgeons $(n=14)$, nurses $(n=4)$, occupational therapists $(n=2)$ and physiotherapist $(\mathrm{n}=1)$.

Non-medical $(\mathrm{n}=23 ; 40 \%)$ and medical professionals $(\mathrm{n}=19 ; 33 \%)$ were generally spending less than one hour on wheelchair assessment while the rest spent more time than that (non-medical;n=13;23\%, medical;n=2;4\%) $\left(\mathrm{x}^{2}=4.83 ; \mathrm{df}=1 ; \mathrm{p}=0.028\right)$.

In 14 cases $(25 \%)$ the latest wheelchair assessment was done in the participants' home, followed by a hospital $(n=13 ; 23 \%)$, in the office of the vendor/supplier $(n=12 ; 21 \%)$, rehabilitation centre $(n=8 ; 14 \%)$ and other $(n=10 ; 18 \%)$. Most $(n=38 ; 67 \% ;)$ attended the wheelchair assessment alone, rather than together with a carer/family member, and were predominantly satisfied with wheelchair assessment (n=51;90\%) As shown in table 4, non-medical profes-

TABLE 3

IMPORTANT ACTIVITIES OF EVERYDAY LIVING MARKED BY PARTICIPANTS*

\begin{tabular}{|c|c|c|c|c|}
\hline \multirow[t]{2}{*}{ Activities of everyday living } & \multicolumn{2}{|c|}{ Satisfied with wheelchair dimensions } & \multicolumn{2}{|c|}{ Satisfied with wheelchair comfort } \\
\hline & $\begin{array}{l}\text { Performing / } \\
\text { Not performing } \\
\mathrm{n}(\%)\end{array}$ & $x^{2} ; d f ; p$-values & $\begin{array}{c}\text { Performing / } \\
\text { Not performing } \\
n(\%)\end{array}$ & $x^{2} ; d f ; p$-values \\
\hline Bathing / showering & $53(63.1) / 27(32.1)$ & $X^{2}=7.18 ; d f=1 ; p=0.016$ & $43(62.3) / 22(31.9)$ & $X^{2}=7.02 ; d f=1 ; p=0.017$ \\
\hline Bladder / bowel management & $38(45.2) / 20(23.8)$ & $X^{2}=27.45 ; \mathrm{df}=1 ; p<0.001$ & $40(58) / 16(23.2)$ & $X^{2}=17.77 ; \mathrm{df}=1 ; \mathrm{p}<0.001$ \\
\hline Intimate hygiene & $46(54.8) / 35(41.7)$ & $\mathrm{X}^{2}=3.76 ; \mathrm{df}=1 ; \mathrm{p}=0.089$ & $42(60.9) / 24(34.8)$ & $\mathrm{X}^{2}=4.87 ; \mathrm{df}=1 ; \mathrm{p}=0.056$ \\
\hline Transfers & $53(63.1) / 25(29.8)$ & $X^{2}=11.04 ; d f=1 ; p=0.002$ & $46(66.7) / 19(27.5)$ & $\mathrm{X}^{2}=8.49 ; \mathrm{df}=1 ; \mathrm{p}=0.010$ \\
\hline Donning / doffing & $51(60.7) / 29(34.5)$ & $\mathrm{X}^{2} 2.42 ; \mathrm{df}=1 ; \mathrm{p}=0.152$ & $49(71) / 17(24.6)$ & $X^{2}=2.40 ; d f=1 ; p=0.182$ \\
\hline Food preparation & $26(31) / 50(59.5)$ & $X^{2}=3.96 ; d f=1 ; p=0.054$ & $29(42) / 27(39.1)$ & $\mathrm{X}^{2}=8.34 ; \mathrm{df}=1 ; \mathrm{p}=0.004$ \\
\hline Eating & $63(75) / 18(21.4)$ & $x^{2}=9.33 ; d f=1 ; p=0.014$ & $53(76.8) / 13(18.8)$ & $\mathrm{X}^{2}=3.72 ; \mathrm{df}=1 ; \mathrm{p}=0.117$ \\
\hline Grocery shopping & $18(21.4) / 53(63.1)$ & $x^{2}=1.95 ; \mathrm{df}=1 ; p=0.280$ & $27(39.1) / 31(44.9)$ & $X^{2}=3.05 ; d f=1 ; p=0.104$ \\
\hline Paid work & $23(27.4) / 43(51.2)$ & $\mathrm{X}^{2}=8.63 ; \mathrm{df}=1 ; \mathrm{p}=0.002$ & $20(29) / 26(37.7)$ & $\mathrm{X}^{2}=14.08 ; \mathrm{df}=1 ; \mathrm{p}<0.001$ \\
\hline Unpaid work / volunteering & $17(20.2) / 34(40.5)$ & $\mathrm{X}^{2}=8.51 ; \mathrm{df}=1 ; \mathrm{p}=0.003$ & $19(27.5) / 23(33.3)$ & $x^{2}=11.10 ; d f=1 ; p=0.001$ \\
\hline Attending school / college & $6(7.1) / 23(27.4)$ & $\mathrm{X}^{2}=8.85 ; \mathrm{df}=1 ; \mathrm{p}=0.006$ & 7 (10.1) / 19 (27.5) & $X^{2}=9.56 ; d f=1 ; p=0.004$ \\
\hline Doing household chores & $21(25) / 30(35.7)$ & $\mathrm{X}^{2}=12.42 ; \mathrm{df}=1 ; \mathrm{p}<0.001$ & $26(37.7) / 21(30.4)$ & $\mathrm{X}^{2}=13.28 ; \mathrm{df}=1 ; \mathrm{p}<0.001$ \\
\hline Taking care of a family member & $15(17.9) / 62(73.8)$ & $\mathrm{X}^{2}=1.66 ; \mathrm{df}=1 ; \mathrm{p}=0.342$ & $18(26.1) / 42(60.9)$ & $X^{2}=3.65 ; d f=1 ; p=0.099$ \\
\hline Taking care of a pet & $8(9.5) / 42(50)$ & $X^{2}=0.91 ; d f=1 ; p=0.513$ & $13(18.8) / 22(31.9)$ & $X^{2}=7.76 ; d f=1 ; p=0.009$ \\
\hline Visiting family / friends & $36(42.9) / 41(18.8)$ & $x^{2}=0.03 ; d f=1 ; p=1.000$ & $34(49.3) / 27(39.1)$ & $x^{2}=2.67 ; d f=1 ; p=0.140$ \\
\hline
\end{tabular}

* $\mathrm{Chi}^{2}$-test for independence ("Important activities of everyday living" against "Satisfied with wheelchair dimensions" and "Satisfied with wheelchair comfort") - Fisher's Exact Test and Bonferroni correction done 
TABLE 4

ASSESSMENT AREAS COVERED BY NON-MEDICAL AND MEDICAL PROFESSIONALS (N=57)

\begin{tabular}{|c|c|c|}
\hline Assessment areas covered during wheelchair assessment & $\begin{array}{c}\text { Asked by non-medical professionals / } \\
\text { Asked by medical professionals } \\
\text { n (\%) }\end{array}$ & $\begin{array}{c}\mathrm{X}^{2} ; \mathrm{df} \\
\mathrm{p} \text {-values* }\end{array}$ \\
\hline Wheelchair type & $31(79,5) / 8(20.5)$ & $\mathrm{X}^{2}=14.15 ; \mathrm{df}=1 ; \mathrm{p}<0.001$ \\
\hline Choice of seat cushions & $31(83.8) / 6(16.2)$ & $\mathrm{X}^{2}=19.28 ; \mathrm{df}=1 ; \mathrm{p}<0.001$ \\
\hline Parts of the body that will be used to operate a wheelchair & $26(81.3) / 6(18.8)$ & $\mathrm{X}^{2}=10.26 ; \mathrm{df}=1 ; \mathrm{p}=0.001$ \\
\hline Releasing pressure while seating & $25(86.2) / 4(13.8)$ & $\mathrm{X}^{2}=13.47 ; \mathrm{df}=1 ; \mathrm{p}<0.001$ \\
\hline Muscle tone and muscle power & $20(87) / 3(13)$ & $X^{2}=9.38 ; d f=1 ; p=0.002$ \\
\hline $\begin{array}{l}\text { Wheelchair option preferences such as reclining feature, } \\
\text { tilt-in-space, seat elevator, standing frame etc. }\end{array}$ & $19(90.5) / 2(9.5)$ & $X^{2}=10.66 ; \mathrm{df}=1 ; p=0.001$ \\
\hline Transportation possibilities in local community & $18(85.7) / 3(14.3)$ & $\mathrm{X}^{2}=7.27 ; \mathrm{df}=1 ; \mathrm{p}=0.007$ \\
\hline Productive activities & $17(85) / 3(15)$ & $\mathrm{X}^{2}=6.31 ; \mathrm{df}=1 ; \mathrm{p}=0.012$ \\
\hline Leisure activities & $16(88.9) / 2(11.1)$ & $x^{2}=7.48 ; \mathrm{df}=1 ; p=0.006$ \\
\hline Transfers & $21(67.7) / 10(32.3)$ & $\mathrm{X}^{2}=0.61 ; \mathrm{df}=1 ; \mathrm{p}=0.433$ \\
\hline Social activities & $14(93.3) / 1(6.7)$ & $x^{2}=7.96 ; d f=1 ; p=0.005$ \\
\hline Indoor and outdoor mobility & $20(66.7) / 10(33.3)$ & $\mathrm{X}^{2}=0.33 ; \mathrm{df}=1 ; \mathrm{p}=0.563$ \\
\hline Past or current changes on skin & $14(51.9) / 13(48.1)$ & $X^{2}=2.81 ; d f=1 ; p=0.093$ \\
\hline Self care activities & $12(75) / 4(25)$ & $\mathrm{X}^{2}=1.34 ; \mathrm{df}=1 ; \mathrm{p}=0.247$ \\
\hline Toileting, bladder and bowel management & $14(82.4) / 3(17.6)$ & $\mathrm{X}^{2}=3.83 ; \mathrm{df}=1 ; \mathrm{p}=0.050$ \\
\hline Showering/bathing & $12(75) / 4(25)$ & $\mathrm{X}^{2}=1.34 ; \mathrm{df}=1 ; \mathrm{p}=0.247$ \\
\hline Medical history & $13(38.2) / 21(61.8)$ & $\mathrm{X}^{2}=22.49 ; \mathrm{df}=1 ; \mathrm{p}<0.001$ \\
\hline Motivation & $8(30.8) / 18(69.2)$ & $X^{2}=21.55 ; d f=1 ; p<0.001$ \\
\hline Cognition/perception/memory & $7(29.2) / 17(70.8)$ & $X^{2}=20.58 ; d f=1 ; p<0.001$ \\
\hline Pain & $10(37) / 17(63)$ & $X^{2}=15.04 ; \mathrm{df}=1 ; \mathrm{p}<0.001$ \\
\hline Sensations & $12(44.4) / 15(55.6)$ & $\mathrm{X}^{2}=7.72 ; \mathrm{df}=1 ; \mathrm{p}=0.005$ \\
\hline
\end{tabular}

* $\mathrm{Chi}^{2}$-test for independence ("Assessment areas covered during wheelchair assessment" against "Asked by non-medical professionals /Asked by medical professionals") - Fisher's Exact Test and Bonferroni correction done

sionals focused more on wheelchair related areas and activities of everyday living, while medical professionals assessed more medical areas. Participants who were assessed only by non-medical professionals ( $n=36 ; 61 \%$ ), have responded feeling safer in using their wheelchairs when compared to those participants who were assessed by medical professionals $(\mathrm{n}=21 ; 37)\left(\mathrm{x}^{2}=22.26 ; \mathrm{df}=1 ; \mathrm{p}<0.001\right)$.

\section{Wheelchair selection process}

Forty-nine participants (86\%) were satisfied with the wheelchair selection process. Participants supported by non-medical professionals $(\mathrm{n}=36 ; 63 \%)$ felt more included in the wheelchair selection process compared to persons supported by medical staff. $\left(x^{2}=20.79 ; d f=1 ; p<0.001\right)$. Participants involved in both wheelchair assessment and wheelchair selection process, were more satisfied with respect to comfort of their wheelchairs $\left(n=50 ; 88 \% ; x^{2}=14.05 ; \mathrm{df}=1\right.$ $; \mathrm{p}<0.001)$ and dimensions such as size $\left(\mathrm{n}=42 ; 74 \% ; \mathrm{X}^{2}=7.29\right.$; $\mathrm{df}=1 ; \mathrm{p}=0.007)$, width $\left(\mathrm{n}=38 ; 67 \% ; \mathrm{X}^{2}=10.84 ; \mathrm{df}=1 ; \mathrm{p}=0.001\right)$, depth $\left(n=46 ; 81 \% ; x^{2}=4.27 ; d f=1 ; p=0.039\right)$ and the ease of ad- justment of wheelchairs $\left(\mathrm{n}=39 ; 70 \% ; \mathrm{X}^{2}=8.63 ; \mathrm{df}=1 ; \mathrm{p}=0.003\right)$ than participants not involved in both processes.

\section{Trial equipment, wheelchair delivery and follow-up}

Only, a minority ( $\mathrm{n}=30 ; 29 \%)$ had a chance to try one or more wheelchair configurations during wheelchair selection process and even fewer $(n=23 ; 77 \%)$ received the same wheelchair they have had a chance to try. These 30 participants who had received the same wheelchair as the one they tried, were more satisfied in terms of functionality/ effectiveness $(n=23 ; 77 \%)\left(x^{2}=7.04 ; d f=1 ; p=0.008\right)$ and durability $(\mathrm{n}=21 ; 70 \%),\left(\mathrm{x}^{2}=4.50 ; \mathrm{df}=1 ; \mathrm{p}=0.034\right)$ of their wheelchair than the participants who had not received the same wheelchair they had a chance to try. Most of these individuals $(n=24 ; 80 \%)$ were satisfied with the equipment trial.

Out of 104 participants, in most of the cases new wheelchairs were delivered to participant's home $(n=54 ; 52 \%)$, at the hospital ( $n=17 ; 16 \%)$, the office of the vendor/supplier 
TABLE 5

AREAS THAT WERE COVERED DURING WHEELCHAIR EDUCATION AND WHEELCHAIR SKILLS TRAINING (N=45)

\begin{tabular}{|c|c|c|}
\hline $\begin{array}{l}\text { Assessment areas covered during wheelchair education and } \\
\text { wheelchair skills training }\end{array}$ & $\begin{array}{c}\text { Done by non-medical professionals } \\
\text { / Done by medical professionals / } \\
\text { Done by other } \\
\text { n (\%) }\end{array}$ & $\begin{array}{c}\chi^{2} ; \mathrm{df} ; \\
\text { p-values* }\end{array}$ \\
\hline Body positioning and body mechanics & $12(54.5) / 9(40.9) / 1(4.5)$ & $\mathrm{X}^{2}=13.29 ; \mathrm{df}=2 ; \mathrm{p}=0.002$ \\
\hline Folding the wheelchair & $12(46.2) / 11(42.3) / 3(11.5)$ & $\mathrm{X}^{2}=7.22 ; \mathrm{df}=2 ; \mathrm{p}=0.021$ \\
\hline Wheelchair warranty & $10(71.4) / 3(21.4) / 1(7.1)$ & $X^{2}=15.43 ; \mathrm{df}=2 ; p=0.001$ \\
\hline Positioning/adjustment of seat cushion & $9(64.3) / 4(28.6) / 1(7.1)$ & $X^{2}=10.58 ; d f=2 ; p=0.008$ \\
\hline Pressure redistribution & $6(66.7) / 3(33.3) / 0$ & $\mathrm{X}^{2}=7.37 ; \mathrm{df}=2 ; \mathrm{p}=0.039$ \\
\hline Removal/adjustment of the backrest & $6(66.7) / 3(33.3) / 0$ & $\mathrm{X}^{2}=7.37 ; \mathrm{df}=2 ; \mathrm{p}=0.039$ \\
\hline Removing the tires & $2(66.7) / 1(33.3) / 0$ & $\mathrm{X}^{2}=2.10 ; \mathrm{df}=2 ; \mathrm{p}=0.437$ \\
\hline Doing transfers & $4(18.2) / 17(77.3) / 1(4.5)$ & $X^{2}=14.54 ; \mathrm{df}=2 ; \mathrm{p}=0.001$ \\
\hline Pushing the wheelchair & $6(20) / 15(50) / 9(30)$ & $X^{2}=8.09 ; d f=2 ; p=0.016$ \\
\hline Entering and exiting the elevator & $1(20) / 4(80) / 0$ & $\mathrm{X}^{2}=2.46 ; \mathrm{df}=2 ; \mathrm{p}=0.481$ \\
\hline Entering and existing through the door & $2(22.2) / 5(55.6) / 2(22.2)$ & $\mathrm{X}^{2}=0.41 ; \mathrm{df}=2 ; \mathrm{p}=0.894$ \\
\hline Turning wheelchair on its axis & $3(33.3) / 5(55.6) / 1(11.1)$ & $\mathrm{X}^{2}=0.56 ; \mathrm{df}=2 ; \mathrm{p}=0.894$ \\
\hline $\begin{array}{l}\text { Overcoming obstacles such as the walkway, the threshold of the } \\
\text { door etc. }\end{array}$ & $2(25) / 4(50) / 2(25)$ & $\mathrm{X}^{2}=0.24 ; \mathrm{df}=2 ; \mathrm{p}=1.000$ \\
\hline Using the wheelchair brakes & $5(19.2) / 14(53.8) / 7(26.9)$ & $\mathrm{X}^{2}=4.57 ; \mathrm{df}=2 ; \mathrm{p}=0.131$ \\
\hline Cleaning the wheelchair & $4(40) / 5(50) / 1(10)$ & $\mathrm{X}^{2}=0.97 ; \mathrm{df}=2 ; \mathrm{p}=0.661$ \\
\hline Tire maintenance such as pressure adjustments & $3(37.5) / 4(50) / 1(12.5)$ & $\mathrm{X}^{2}=0.40 ; \mathrm{df}=2 ; \mathrm{p}=1.000$ \\
\hline Removal/adjustments of the safety belts/harnesses & $3(42.9) / 4(57.1) / 0$ & $\mathrm{X}^{2}=2.14 ; \mathrm{df}=2 ; \mathrm{p}=0.423$ \\
\hline Removal/adjustment of the footrest & $5(45.5) / 6(54.5) / 0$ & $X^{2}=3.97 ; d f=2 ; p=0.116$ \\
\hline Removal/adjustment of the armrest & $4(40) / 6(60) / 0$ & $\mathrm{X}^{2}=3.22 ; \mathrm{df}=2 ; \mathrm{p}=0.205$ \\
\hline Battery charging and maintenance & $2(40) / 3(60) / 0$ & $X^{2}=1.41 ; d f=2 ; p=0.686$ \\
\hline $\begin{array}{l}\text { Driving a wheelchair on slopping surfaces including the ramps, } \\
\text { downhill/uphill }\end{array}$ & $3(42.9) / 3(42.9) / 1(14.3)$ & $\mathrm{X}^{2}=0.56 ; \mathrm{df}=2 ; \mathrm{p}=0.868$ \\
\hline Driving a wheelchair over unpaved roads & $2(40) / 2(40) / 1(20)$ & $\mathrm{X}^{2}=0.23 ; \mathrm{df}=2 ; \mathrm{p}=0.841$ \\
\hline Driving a wheelchair while using a private or public transport & $3(50) / 3(50) / 0$ & $\mathrm{x}^{2}=2.18 ; \mathrm{df}=2 ; \mathrm{p}=0.371$ \\
\hline Removal/adjustment of the anti-tippers & $3(50) / 3(50) / 0$ & $\mathrm{X}^{2}=2.18 ; \mathrm{df}=2 ; \mathrm{p}=0.371$ \\
\hline Wheelchair handles adjustment & $3(50) / 3(50) / 0$ & $\mathrm{X}^{2}=2.18 ; \mathrm{df}=2 ; \mathrm{p}=0.371$ \\
\hline
\end{tabular}

${ }^{*} \mathrm{Chi}^{2}$-test for independence ("Assessment areas covered during wheelchair education and wheelchair skills training” against "Done by non-medical professionals/Done by medical professionals/Done by other") - Fisher's Exact Test and Bonferroni correction done

$(\mathrm{n}=12 ; 12 \%)$, or the rehabilitation centre $(\mathrm{n}=8 ; 8 \%)$ while the rest were delivered somewhere else $(n=13 ; 13 \%)$. Only 7 participants received a follow-up, leaving 97 participants without follow-up regarding wheelchair use, skills, safety and maintenance. Medical professionals mostly did those follow-ups.

\section{Wheelchair education and skills training}

Only 45 participants (43\%) received professional wheelchair education and wheelchair skills training, i.e. in 22 cases this was provided by medical professionals, in 14 cases by non-medical professionals and in nine participants by other person. Areas that were covered during wheelchair education and wheelchair skills training are presented in table 5 .

In 40 cases (89\%), participants were very or somewhat satisfied, while 5 persons (11\%) were not satisfied with the wheelchair education and wheelchair skills training. No difference was found in satisfaction levels between participants met by medical compared to non-medical professionals $\left(x^{2}=0.36 ; d f=2 ; p=0.833\right)$. Participants taking part in these two processes felt safer using their wheelchairs compared to the participants not involved $(n=23 ; 51 \%)$ $\left(x^{2}=6.13 ; \mathrm{df}=1 ; \mathrm{p}=0.013\right)$. In 18 cases wheelchair education and wheelchair skills training was done in the participants' adapted dwellings. Most participants received wheelchair 
education and wheelchair skills training $(n=26 ; 58 \%)$ lasting less than 15 minutes, 12 participants between $15-60$ minutes and 7 participants for more than one hour. Medical professionals spent mostly less than 15 minutes on these processes, while non-medical professionals mainly spent between 30-60 minutes on same processes.

\section{Fitting process, repairs and knowledge about availability of wheelchair services in community}

In most cases, i.e., 60 participants (58\%) the assessors did not carry out the fitting process upon delivery to user. Twenty-six participants got their wheelchairs fitted by non-medical professionals, nine participants respectively by medical professionals or other persons such as children, partners, caregivers, friends or somebody else. Out of those 44 participants, 38 participants were satisfied with the fitting process.

Eighty-two participants (78\%) had to make repairs with their wheelchairs, most often done by laypersons (54 cases) compared to 28 cases repaired by non-medical professionals. Nevertheless, majority of participants $(n=65 ; 79 \%)$ were satisfied with the repairs done.

Table 6 presents participants' knowledge about availability of wheelchair services in their local community.

\section{Home modifications}

In total sample, 62 participants (60\%) stated that their home was modified to allow free movement for their wheelchairs. Participants who received home modifications felt safer $(p<0.001)$ using wheelchairs indoor than those who did not have such adaptations (Table 7). Installation of $\operatorname{ramp}(\mathrm{n}=35 ; 34 \%)$, levelling the entrance to the ground floor $(n=13,13 \%)$ and installing the external elevator $(n=6 ; 6 \%)$ were most common modifications. In Zagreb, the capital city of Croatia, the majority of participants' dwellings were not modified for the safe wheelchair use. In total, 42 participants (40\%) did not receive any home modifications to support wheelchair use, resulting in facing challenges with the existing stairs and inappropriate ramp.

\section{Participants' comments}

Twenty-one participants gave additional comments at end of questionnaire. Fifteen participants criticised the lack of wheelchair centres in their local community. Negative opinions were also given about incomplete wheelchair assessment processes and insufficient wheelchair skills training, extremely poorly organized follow-ups and physical barriers at home.

\section{Discussion}

In the present study, majority of participants were satisfied with possibilities to adjust wheelchair parts and in general with the wheelchairs functionality. Similar findings can be found in other studies ${ }^{28,29}$ except for the comfort and wheelchair adjustability ${ }^{30}$. Several studies ${ }^{8,31}$ indicated that level of participants' satisfaction did not depend on sex, age or aetiology, which was confirmed by our results. However, participants who had used wheelchairs for more than 5 years, rather than a shorter time, were more satisfied with the comfort of their wheelchairs. This can possibly be explained by the process of accommodation where the body if not properly positioned or placed in the wheelchair, can adapt to a new structural environment and alter the participant's cognitive/reasoning aspects after which the participant starts accepting faults of the wheelchair and/or accessories and starts accommodating to it ${ }^{1,2,6,32,33}$. Additionally, approximately half of our par-

TABLE 6

KNOWLEDGE ABOUT AVAILABILITY OF WHEELCHAIR SERVICES IN THE PARTICIPANTS' LOCAL COMMUNITY

\begin{tabular}{lcc}
\hline In my community I know where to: & \multicolumn{2}{c}{ Home modifications N=104 } \\
\cline { 2 - 3 } & Know n (\%) & Don't know n (\%) \\
\hline .. ask for a new wheelchair & $85(82)$ & $19(18.3)$ \\
$\ldots$ ask for wheelchair repairs/maintenance & $56(54)$ & $48(46.2)$ \\
$\ldots$... ask for additional evaluations/second opinions & $24(23.1)$ & $80(77)$ \\
$\ldots$ seek wheelchair support groups & $26(25)$ & $78(75)$ \\
\hline
\end{tabular}

TABLE 7

Home modifications

\begin{tabular}{|c|c|c|c|}
\hline \multirow[t]{3}{*}{ Place of residence } & \multicolumn{2}{|c|}{ Home modifications $\mathrm{N}=104$} & \multirow{3}{*}{$\begin{array}{c}\chi^{2} ; \mathrm{df} ; \\
\text { p-values }\end{array}$} \\
\hline & Done $(\mathrm{n}=62)$ & Not done $(n=42)$ & \\
\hline & N (\%) & $\mathrm{N}(\%)$ & \\
\hline Zagreb (the capital city of Croatia) & $12(11.5)$ & $28(27)$ & \multirow{2}{*}{$\begin{array}{c}\mathrm{X}^{2}=23.68 ; \mathrm{df}=1 ; \\
\mathrm{p}<0.001\end{array}$} \\
\hline Other cities, towns and villages & $50(48.1)$ & $14(13.5)$ & \\
\hline
\end{tabular}


ticipants had a wheelchair assessment which would suggest that the rest were given random wheelchairs.

Although the majority of participants in the present study were satisfied with all wheelchair features, more than half of the participants stated to have injured themselves when using a wheelchair, while only one-third of the participants mentioned reasons for not feeling safe. Poorly adjusted or missing wheelchair accessories (i.e. a cushion, belt), heavy wheelchair, slippery upholstery, sliding down in wheelchair and malfunctioning of wheelchair parts (i.e. the brakes, wheels, armrests) were found to be the top reasons causing feelings of insecurity when using a wheelchair as well as causes of wheelchair injuries. Similar findings can be found in the study done by Perks et al. ${ }^{34}$ where more than half of all participants felt their wheelchairs did not properly meet their needs, also causing inefficient wheelchair propulsion and decreased involvement in the activities of everyday living.

We also found that participants who were satisfied with the dimensions of their wheelchairs and who felt comfortable when sitting in their wheelchairs were more involved in the activities of everyday living such as bathing/ showering, bladder/bowel management, doing transfers, and other activities. Similar studies ${ }^{28,29}$ have also shown that satisfaction with the wheelchairs, such as dimensions, comfort, simplicity to use a wheelchair and other features led to more active lifestyles. Benefits of active lifestyle for wheelchair users as for humans in general are numerous; for example independence in the activities of everyday living, increased socialisation and participation in the community through indoor and outdoor mobility, but also health prevention through facilitating bodily functions such as breathing, digestion and other ${ }^{1,2,6,35,36}$. It is well known that self-care, productive and leisure activities are crucial for the health and well-being of any client $^{19,37}$. Thus, it is important to offer assessments and interventions to wheelchair users aiming to maintain, enable or increase participation in activities.

Although many participants in the present study were satisfied with the wheelchair characteristics, their performance of activities of everyday living were limited although these activities were of great importance for them. Unfortunately, it is unclear from our findings if participants had the underlying abilities to perform these activities. However, the lack of occupational therapists in the wheelchair service centres, may offer one potential explanation to limitations in the activities of everyday living among participants. All wheelchair service team members, particularly occupational therapists, should provide client-centred interventions directed towards the wheelchair users and their family members/carers in their living and working environment, support activities and participation.

In the presented study, participants with whom the wheelchair assessment and wheelchair selection process were done, reported more satisfaction with the comfort and dimensions of their delivered wheelchairs as well as with the easiness to adjust certain wheelchair parts. This is to be expected as when wheelchair users are included in these processes, and are done thoroughly, the final out- come should always be satisfaction with the wheelchair characteristics. Unfortunately, almost half of the participants in the present study were not involved in those processes. This is another major problem identified in the present study as wheelchairs should never be given to users without prior inclusion of the users in the assessment process. Participants were either assessed by non-medical or medical professionals, mostly in their homes and they were predominantly satisfied with both procedures. However, they responded feeling safer in using their wheelchairs after receiving the assessment from non-medical professionals. The reasons for that could be in the duration and areas covered during the wheelchair assessment but also in the client-centred approach in which participants were not always holistically assessed by medical professionals and considered as experts on their own situation. Non-medical professionals spent more time with wheelchair users than medical professionals. Apart from that their job is related to selling the wheelchairs, it is likely that they were more educated in the wheelchair assessment process than medical professionals, thus they required more time to complete the evaluation.

The reasons for such result might be in a more clientcentred approach that the non-medical professionals use, especially in terms of allowing the users to voice their concerns and were found to spent on average more time with the clients. Aside from this, non-medical professionals are also involved with retail which could mean they are more familiar with the technical aspects and therefore could offer the clients more information during the assessment process. This is also shown in the results where nonmedical professionals were found to assess more areas related to wheelchair type and option preferences, choice of seat cushions, pressure redistribution when sitting, parts of the body that will be used to operate a wheelchair as well as transportation possibilities within the participant's local community. When compared to medical professionals they were also asking more about activities of everyday living, meaning that they used a more client-centred approach (Table 4). Every wheelchair that is prescribed to a wheelchair user should compensate for the decreased or lost functions and allow the user to be as active as possible in the activities of everyday living. It should also increase or maintain user's body functions and prevent any future deterioration, thus proper wheelchair assessment and wheelchair selection process is crucial ${ }^{9,17,24}$. Additionally, more than half of the participants attended the wheelchair assessment and wheelchair selection process alone, that is without a carer or a family member. This is not in accordance with the international guidelines that clearly state the wheelchair user's involvement in both processes together with his/her family members/carers is crucial $^{9,14,23,36,38}$.

Interestingly, although the narrow majority of participants were involved in the wheelchair assessment and wheelchair selection process, only 30 percent had a chance to try one or more wheelchairs/configurations before the wheelchair order was placed. After the wheelchair assessment, wheelchair users should be involved in the wheel- 
chair trial period, as this has a very significant role on the user's overall satisfaction. In the present study, most of the wheelchairs were delivered to client's home. Ideally, every wheelchair delivery should be done at the specialist wheelchair centre with the access to workshop facilities where the proper risk-assessment can be carried out and every modification needed to be done $e^{9,11,24,36}$. Benefits for the wheelchair user when delivering a wheelchair to his/ her home may include immediate testing of the wheelchair in the wheelchair user's real environment, free delivery or reduced travelling costs, more personal time on disposal and potential alleviation of physical and emotional energy that could be consumed when going to the wheelchairservice centre. On the other hand, delivering a wheelchair to the user's home may significantly affect the quality of care due to the lack of necessary equipment for transfers, availability of wheelchair tools and workshop stations, availability of multidisciplinary team members and potential challenges of properly fitting the wheelchair to the user. It is interesting to note that in the present study participants who had received the same or similar wheelchair as the one they tried, were more satisfied with the easiness to use a wheelchair and durability than the participants with whom only the wheelchair assessment and wheelchair selection process were done or not done at all.

Less than half of all participants received wheelchair education and skills training which is problematic. The user should always be trained in the wheelchair use and in order to increase safety, wheelchair education and wheelchair skills training should be done and reviewed in the wheelchair user's real environment where the wheelchair will be used ${ }^{10}$. Participants who had received wheelchair education and wheelchair skills training responded feeling safer in using their wheelchairs. This is to be expected because proper wheelchair training should have a positive effect on the client's capacity to use the device as well as increase safety ${ }^{1,2,6,9,10}$.

The majority of participants did not get the wheelchair fitted when the wheelchair was delivered to them, which means that the participants had to adjust the off-the-shelf equipment themselves according to their best knowledge and "feelings" of what is correct. For the rest of the participants the wheelchair was fitted by non-medical professionals or by their children, partners, friends or caregivers. This is a short-coming and a potential safety risk. It is also worth to mention, that the international practice guidelines clearly state that the wheelchair fitting should be done by a competent/skilled professional whenever needed, especially when the new wheelchair is being delivered $^{9,14,23,36,38}$.

As presented in table 7, when compared to the wheelchair users with whom the home modifications were done, less home modifications were made in Zagreb - the capital city of Croatia, than in other cities. It may be due to wheelchair users in smaller cities or villages have much supportive local communities, that is they help each other more frequently or get help from authorities. In Zagreb, the outdoor environment for the wheelchair users has significantly improved in the last decade, however when com- pared to other cities in Croatia, there are still many participants left in the capital city whose indoor environment was not modified for the safe and free wheelchair use.

In total, less than half of the participants had not received any home assessments or modifications that would allow free and safe movement of their wheelchair. Participants, with whom home modifications were done, responded feeling safer when using their wheelchairs. This is to be expected since home modifications are likely to increase wheelchair user's safety as well as independence.

In the present study only few participants were followed up after a wheelchair delivery regarding their wheelchair use, skills, safety and repairs which presents another major problem. According to the international guidelines ${ }^{9,10}$, first follow-ups should be done within first 3 months after a wheelchair delivery and clinical reviews should be done ideally every 6-12 months at a minimum or at any other time when there are significant changes such as in wheelchair user's health. Moreover, when a wheelchair replacement is required or if there are delays between the wheelchair prescription process, funding and wheelchair delivery, follow-ups should be done too ${ }^{9}$.

The lack of follow-ups is a clear disadvantage, since each wheelchair service should have clear guidelines for the frequency of follow-ups as well as different types of reviews such as technical, clinical or multidisciplinary ${ }^{9}$. Every follow-up should include review of the wheelchair user's needs, appropriateness of the wheelchair, fit and use of the current wheelchair as well as the wheelchair users and carer's ongoing education about the use and risks of the equipment ${ }^{9,23,39}$. Having an established follow-up systems are necessary for the wheelchair user's satisfaction level of the prescribed wheelchair as well as for the wheelchair user's health and well-being. The risks of not doing follow-ups are vast, both for the wheelchair user and the wheelchair service team. Some of those risks include lack of obtaining the feedback on the wheelchair user's intervention goals and outcomes, body functions, mobility, participation in the activities of everyday living, carer's challenges, wheelchair operation abilities and safety. Moreover, lack of follow-ups does not allow the wheelchair team members making any wheelchair or environmental adjustments if required, thus keeping a wheelchair user at a risk of injury $^{9,10}$. The international practice guidelines strongly suggest incorporating wheelchair users regular reviews and reassessments within each wheelchair service centre, instead of relying on the wheelchair users to report themselves once the problem arise $e^{9,38}$. Consequently, it is a shortcoming that this was not done by assessors in the present study.

The repairs/maintenance services were not available to all participants in the present study and that represents another disadvantage. It is evident from the results that most of the participants had repairs done by laypersons (family or friends), while minority asked for professional help. Maintenance and repairs are important factors for efficient and safe wheelchair use. The risks of doing the repairs by non-competent laypersons, is to mechanical and/ or electrical failures to the wheelchair ${ }^{40,41}$. Nevertheless, the 
majority of the participants in the present study were satisfied with the repairs done. It is important to emphasize that literature suggest that basic routine maintenance and services e.g. checking tires, breaks and other wheelchair parts for any damages or malfunctions could be done by the wheelchair users and carers. Routine maintenance should increase the life duration of the wheelchair parts and it should be done regularly. However, professionals should carry out advanced services and repairs of any sort $\mathrm{t}^{9,10}$.

In the present study many participants did not know where they could ask for additional wheelchair evaluations/ second opinions, wheelchair support groups and wheelchair repairs/maintenance in their local community. It is evident from the literature that the wheelchair suppliers should always provide details to the wheelchair users about wheelchair assessment, support groups as well as their local wheelchair service maintenance centres ${ }^{9,10}$. The wheelchair assessors must ensure that the manufacturer's information regarding the wheelchair use and maintenance is always provided to the user ${ }^{10}$. Literature suggest that in addition to the regular reviews that should be carried out by the wheelchair users (if possible), carers and the wheelchair service team, there should be a defined mechanism in the local community for maintaining and repairing any type of the wheelchair seating system from the skilled professionals ${ }^{9,10,42}$. All repairs should be prioritised and risk assessments should be done by competent engineering staff ${ }^{9}$. Additional comments from participants show that majority of the comments were negative towards the lack of wheelchair services. Thus, there is a need for future research in this area as well as improvements, to promote health and well-being of the wheelchair users.

\section{Strengths and Limitations}

The results of the study present a first look into the wheelchair users' satisfaction with wheelchair services in Croatia, however study limitations need to be addressed.

By using an online survey there is no way to calculate a true response rate and there is also a chance that some of participants gave more sociably desirable answers which can lead to some bias. However, through the study design using an online survey it was possible to generate data relevant to answer the study purpose.

The overrepresentation of male participants with spinal cord injury may prevent the generalizability of the results. However, this reflects the target population ${ }^{43}$, since more men than women between the 18-65 years of age have physical disability in Croatia with the most prevalent cause of disability being impairments of the locomotor system.

The study participants were recruited via online platform, which means that a true response rate cannot be calculated. Also the questionnaire used was not validated. Due to the nature of the questions it is possible that some participants gave answers they felt were more desirable which may contribute to some data distortion.

Further research should focus on longitudinal studies that may provide insights into causal relationship between wheelchair satisfaction and activities of daily living.

\section{Conclusion}

The results of the present study indicate that satisfaction with the wheelchair dimensions and comfort allowed participants to become more involved in their important activities of everyday living and increased their feelings safety when using a wheelchair. Likewise, when the participants were included in the wheelchair selection process and when wheelchair skills training were done with them, they acknowledged feeling safer when using their wheelchairs. Despite these positive characteristics, many participants were not included in some or all parts of the wheelchair process, which reflected negatively on their performance in the activities of everyday living, feelings of safety while using a wheelchair and satisfaction with the wheelchair characteristics and wheelchair services. Even though many participants were satisfied with the wheelchair characteristics and wheelchair services, the findings shed light on the lack of established guidelines and practices in the wheelchair service centres in Croatia regarding the wheelchair assessment, wheelchair selection process and trial equipment, wheelchair skills training, wheelchair delivery and fitting process, repairs and follow-ups. Further research should be done within these areas with the assessors themselves together with proper education in posture management and mobility assessment. Moreover, occupational therapists should be included more in all wheelchair phases and follow evidencebased practice guideline, especially wheelchair skills training in order to improve and maintain the needed skills for everyday living. Educational efforts should be directed towards existing wheelchair services to increase their performance and wheelchair users' well-being.

\section{Conflict of Interest}

All Authors declare no conflict of interest.

\section{Acknowledgments}

The Author would like to thank the Croatian Paraplegic and Tetraplegic Alliance for the help with the participant recruitment.

\section{R E F E R E N C E S}

1. BATAVIA M, The Wheelchair Evaluation - A Clinician's Guide, (Jones and Bartlett Publishers, Canada 2010). — 2. POPE PM, Severe and Complex Neurological Disability - Management of the Physical Condition
(Elsevier Ltd, Philadelphia, 2007). — 3. COOPER RA, Rehabilitation Engineering Applied to Mobility and Manipulation (IOP Publishing Ltd, London, 1995). - 4. MAYALL JK, DESHARNAIS G, Positioning in a 
Wheelchair: A Guide for Professional Caregivers of the Disabled Adult second edition, (NJ: Slack Thorofare, 1995). - 5. MC.DONALD RL, Wheelchairs: posture and mobility. In: CURTIN M, MOLINEUX M, SUPYK-MELLSON J (Eds) Occupational Therapy and Physical Dysfunction - Enabling Occupation (Churchill Livingstone, Philadelphia, 2010). -6. ZOLLARS JA, Special Seating: An Illustrated Guide - Revised Edition, (Prickly Pear Publications, Albuquerque, 2010). - 7. ZACHARKOW D, Wheelchair Posture and Pressure Sores, (Charles C Thomas, Illinois, 1984). - 8. DE GROOT S, POST MW, BONGERS-JANSSEN HM, BLOEMEN-VRENCKEN JH, VAN DER WOUDE LH, Spinal Cord, 49 (2011) 560-5. DOI: 10.1038/sc.2010.150. — 9. BRITISH SOCIETY OF REHABILITATION MEDICINE, Specialised Wheelchair Seating National Clinical Guidelines (British Society Of Rehabilitation Medicine, London, 2004). - 10. ENABLENSW, Guidelines for the prescription of a seated wheelchair or mobility scooter for people with a traumatic brain injury or spinal cord injury 2011, Accessed 20.02.2017. Available from: https://www. aci.health.nsw.gov.au/_data/assets/pdf_file/0003/167286/Guidelines-onWheelchair-Prescription.pdf. - 11. ROYAL COLLEGE OF PHYSICIANS, The provision of wheelchairs and special seating - guidance for purchasers and providers: report of a working group of the Royal College of Physicians, (RCP, London, 1995). - 12. POPE PM, Physiotherapy, 12 (1992) 896-903. DOI: https://doi.org/10.1016/S0031-9406(10)60494-4 .13. CUTTER N, BLAKE D, Physical Medicine Rehabilitation, 1 (1997) 107-32. - 14. MCELDERRY F, NICHOLSON J, FIELDS M, Specialised Wheelchair Seating National Clinical Guidelines for good practice, (College of Occupational Therapy, London 2000). - 15. ANGELO J, BUNING ME, SCHMELER M, DOSTER S, Am J Occup Ther, 51 (1997) 916-20. DOI: DOI: 10.5014/ajot.51.10.916. - 16. MORTENSON WB, MILLER WC, CanJ Occup Ther, 75(2008) 167-75. DOI: 10.1177/000841740807500308. — 17. SAMUELSSON K, WRESSLE E, Disabil Rehabil, 30 (2008)551-8. DOI: 10.1080/09638280701355777. — 18. TURNER BJ, OWNSWORTH TL, TURPIN M, FLEMING JM, GRIFFIN J, Aust Occup Ther J, 55 (2008) 96-107. DOI: 10.1111/j.1440-1630.2007.00660.x. - 19. KIELHOFNER G, Model Of Human Occupation - theory and application, (Williams \& Wilkins, Baltimore, 2008). - 20. HASTINGS JD, Phys Med Rehabil Clin N Am, 11 (2000) 183-207. PMID: 10680165. - 21. KANYER B, The Journal of Head Trauma Rehabilitation, 7 (1992) 81-93. DOI: DOI: 10.1097/00001199-199209000-00010. — 22. FRENEY D, Rahab Management International, 4 (1995) 155-6. - 23. NICHOLSON J, MCELDERRY F. Assessment and Provision of Special Seating: NAPOT Guidelines for Good Practice (College of Occupational Therapists, London, 2000). - 24.
NATIONAL WHEELCHAIR SERVICE STANDARDS, A consortium, facilitated by the posture and mobility group for England and Wales, (The Posture and Mobility Group for England and Wales, 2004). — 25. SCHERER MJ, CUSHMAN LA, Disabil Rehabil, 23 (2001) 387-93. PMID: 11394589. DOI: DOI: 10.1080/09638280010006665. — 26. SHARMANA, PONTON T, Physiotherapy, 3 (1990) 187-91. DOI: DOI:10.1016/S00319406(10)62170-0. - 27. STEWART CP, Prosthet Orthot Int, 15 (1991) 193-8. DOI: 10.3109/03093649109164288. - 28. CHAN SC, CHAN AP, Occup Ther Int, 14 (2007) 123-43. DOI: 10.1002/oti.228. - 29. GIESBRECHT EM, RIPAT JD, QUANBURY AO, COOPER JE, Disabil Rehabil Assist Technol, 4 (2009) 198-207. DOI: 10.1080/17483100802543205. - 30. VACHON B, WEISS-LAMBROU R, LACOSTE M, DANSEREAU J, Proceedings of the Annual RESNA Conference: Elderly nursing home residents - satisfaction with manual and powered wheelchairs (RESNA Press, Washington DC, 1999). - 31. MARCHIORI C, BENSMAIL D, GAGNON D, PRADON D, J Rehabil Res Dev, 52 (2015) 181-92. DOI:10.1682/JRRD.2014.04.0092. — 32. MERVYN LETTS R, Principles of Seating the Disabled (CRC Press, Florida, 1991). — 33. HAM R, ALDERSEA P, PORTER D, Wheelchair Users and Postural Seating: A Clinical Approach (Churchill Livingstone, United Kingdom, 1998). — 34. PERKS BA, MACKINTOSH R, STEWART CP, BARDSLEY GI, J Rehabil Res Dev, 31 (1994) 297-302. PMID: 7869277. — 35. RUSHMAN C, SHANGALI HG, Wheelchair service guide for low-income countries (Tumani University, Moshi, 2005). - 36. UNITED NATIONS, Convention on the Rights of Persons with Disabilities (United Nations, 2006). - 37. KIELHOFNER G, Conceptual foundations of occupational therapy (F.A. Davis Company, Philadelphia, 2009). - 38. THE AUDIT COMMISSION, Fully Equiped: The provision of equipment to older and disabled people by the NHS and Social Services in England and Wales (Audit Commission, London, 2000). - 39. HEALY A, RAMSEY C, SEXSMITH E, Dev Med Child Neurol. 39 (1997) 706-10. DOI: DOI: 10.1111/j.1469-8749.1997. tb07368.x. - 40. FRANK AO, WARD J, ORWELL NJ, MCCULLAGH C, BELCHER M, Clin Rehabil, 14 (2000) 665-73. DOI: 10.1191/0269215500cr376oa. - 41. MCCLURE LA, BONINGER ML, OYSTER ML, WILLIAMS S, HOULIHAN B, LIEBERMAN JA, COOPER RA, Arch Phys Med Rehabil. 90 (2009) 2034-8. DOI: 10.1016/j. apmr.2009.07.020. - 42. WEISS-LAMBROU R, TREMBLAY C, LEBLANC R, LACOSTE M, DANSEREAU J, Assist Technol, 1 (1999) 43-53. DOI: 10.1080/10400435.1999.10131984. - 43. CROATIAN INSTITUTE OF PUBLIC HEALTH, Report of the Disabled Persons in the Republic of Croatia (Croatian Institute of Public Health, Zagreb, 2017).

\section{ZADOVOLJSTVO KORISNIKA S PROPISANIM INVALIDSKIM KOLICIMA I USLUGOM DOBIVANJA INVALIDSKIH KOLICA U HRVATSKOJ}

\section{S A Ž E T A K}

Autori su pokušali utvrditi zadovoljstvo korisnika invalidskih kolica s izdanim invalidskim kolicima i uslugama izdavanja invalidskih kolica u Hrvatskoj. Istraživanje je provedeno uz pomoć internetske platforme među članovima Hrvatskog paraplegičkog i tetraplegičkog saveza. Ukupno, 104 sudionika je uključeno u analizu koristeći neparametrijsku statistiku. Većina sudionika je izrazila zadovoljstvo sa dimenzijama invalidskih kolica (n=84;81\%), funkcionalnosti ( $n=80 ; 77 \%)$, jednostavnosti korištenja ( $n=81 ; 78 \%)$, udobnosti $(n=69 ; 66 \%)$ i mogućnostima prilagodbe dijelova invalidskih kolica (n=64;62\%). Pojedinci zadovoljni karakteristikama invalidskih kolica su također bili više angažirani u aktivnostima svakodnevnog života od sudionika koji nisu bili zadovoljni. Značajno sudjelovanje je pronađeno u slijedećim aktivnostima: kupanje / tuširanje $\left(x^{2}=7.02 ; \mathrm{df}=1 ; \mathrm{p}=0.017\right)$, transferi $\left(x^{2}=8.49 ; \mathrm{df}=1 ; \mathrm{p}=0.01\right)$, vršenje nužde $\left(x^{2}=17.77 ; \mathrm{df}=1 ; \mathrm{p}<0.001\right)$, pripremanje hrane $\left(\mathrm{x}^{2}=8.34 ; \mathrm{df}=1 ; \mathrm{p}=0.004\right)$, hranjenje $\left(\mathrm{X}^{2}=9.33 ; \mathrm{df}=1 ; \mathrm{p}=0.014\right)$ i obavljanje kućanskih poslova $\left(\mathrm{x}^{2}=13.28 ; \mathrm{df}=1 ; \mathrm{p}<0.001\right)$. Pedest i sedam sudionika $(54.8 \%)$ koji su sudjelovali u procjeni invalidskih kolica izrazili su zadovoljstvo procjenom ( $\mathrm{n}=51 ; 90 \%)$, selekcijom $(\mathrm{n}=49 ; 79 \%)$, isprobavanjem opreme $(\mathrm{n}=24 ; 80 \%)$, edukacijom/treningom vještina $(n=40 ; 89 \%)$ i procesom namještanja $(n=38 ; 86 \%)$ invalidskih kolica. Sudionici koji su sudjelovali u procjeni i treningu vještina bili su sigurniji kod korištenja invalidskih kolica $\left(\mathrm{x}^{2}=6.13 ; \mathrm{df}=1 ; \mathrm{p}=0.013\right)$. Većina sudionika nije znala gdje bi mogla tražiti drugo mišljenje vezano uz procjenu invalidskih kolica (n=80;77\%) ili grupe za podršku korisnika invalidskih kolica $(n=78 ; 75 \%)$. Istraživanje je pokazalo kako zadovoljstvo invalidskim kolicima i uslugom dobivanja invalidskih kolica dovodi do veće uključenosti u aktivnosti svakodnevnog života i povećava osjećaj sigurnosti. 